\title{
Anti-sunward high-speed jets in the subsolar magnetosheath
}

\author{
F. Plaschke ${ }^{1,2}$, H. Hietala ${ }^{3,4}$, and V. Angelopoulos ${ }^{1}$ \\ ${ }^{1}$ Institute of Geophysics and Planetary Physics, and Department of Earth and Space Sciences, \\ University of California Los Angeles, Los Angeles, CA, USA \\ ${ }^{2}$ Space Research Institute, Austrian Academy of Sciences, 8042 Graz, Austria \\ ${ }^{3}$ Space and Atmospheric Physics Group, The Blackett Laboratory, Imperial College, London, SW7 2AZ, UK \\ ${ }^{4}$ Department of Physics, University of Helsinki, Helsinki, Finland \\ Correspondence to: F. Plaschke (fplaschke@igpp.ucla.edu)
}

Received: 22 May 2013 - Revised: 17 September 2013 - Accepted: 27 September 2013 - Published: 31 October 2013

\begin{abstract}
Using 2008-2011 data from the five Time History of Events and Macroscale Interactions during Substorms (THEMIS) spacecraft in Earth's subsolar magnetosheath, we study high-speed jets identified as intervals when the antisunward component of the dynamic pressure in the subsolar magnetosheath exceeds half of its upstream solar wind value. Based on our comprehensive data set of 2859 highspeed jets, we obtain the following statistical results on jet properties and favorable conditions: high-speed jets occur predominantly downstream of the quasi-parallel bow shock, i.e., when interplanetary magnetic field cone angles are low. Apart from that, jet occurrence is only very weakly dependent (if at all) on other upstream conditions or solar wind variability. Typical durations and recurrence times of high-speed jets are on the order of tens of seconds and a few minutes, respectively. Relative to the ambient magnetosheath, high-speed jets exhibit higher speed, density and magnetic field intensity, but lower and more isotropic temperatures. They are almost always super-Alfvénic, often even super-magnetosonic, and typically feature 6.5 times as much dynamic pressure and twice as much total pressure in anti-sunward direction as the surrounding plasma does. Consequently, they are likely to have significant effects on the magnetosphere and ionosphere if they impinge on the magnetopause.
\end{abstract}

Keywords. Magnetospheric Physics (Magnetosheath; Solar wind-magnetosphere interactions)

\section{Introduction}

Within the dayside, subsolar magnetosheath, the plasma dynamic pressure is in general much lower than in the pristine solar wind. Occasionally, however, transient enhancements of the dynamic pressure that stand out from the usual magnetosheath turbulence are observed. If these enhancements are directed toward the magnetopause, their impact on the magnetopause-magnetosphere-ionosphere system can be remarkably strong (see, e.g., Shue et al., 2009; Amata et al., 2011). Some enhancements may be caused by dynamic pressure fluctuations embedded in the solar wind. In the absence of such upstream causes, other generation mechanisms have to be responsible. These mechanisms (i) can result in global or localized dynamic pressure enhancements; (ii) can be related to certain steady upstream solar wind conditions or variations thereof; (iii) can act in the foreshock, at the bow shock, or within the magnetosheath itself.

Lin et al. (1996a, b) modeled the interaction of the bow shock with a rotational discontinuity in the interplanetary magnetic field (IMF) using magnetohydrodynamic (MHD) and hybrid simulation codes. They found that when rotational discontinuities pass the bow shock or interact with back-streaming ions upstream of the bow shock (in the foreshock region; see also Omidi and Sibeck, 2007; Turner et al., 2013), global-scale, transient enhancements in dynamic pressure are produced. These enhancements feature increased densities and boundary tangential flow velocities. Observational evidence of such enhancements has recently been presented by Archer et al. (2012) and Dmitriev and Suvorova (2012). 
Savin et al. (2012) presented a study of the relationship between magnetosheath plasma jets and hot flow anomalies upstream of the bow shock (e.g., Schwartz et al., 2000). The authors argue that hot flow anomalies (obstacles to the solar wind flow) trigger high-speed jets (HSJs) locally to restore solar wind flux balance (see also, Savin et al., 2004, 2008). Because interactions of current sheets in the solar wind with the bow shock are required for hot flow anomaly occurrence (e.g., Schwartz et al., 2000; Omidi and Sibeck, 2007), the work of Savin et al. (2012) again relates the appearance of magnetosheath plasma jets to discontinuities in the IMF.

Another generation mechanism for localized high-speed jets was introduced by Hietala et al. (2009, 2012), who analyzed HSJ observations in the dayside magnetosheath downstream of the quasi-parallel bow shock (steady, radial IMF): previous studies suggest the quasi-parallel shock front to be dynamically formed and reformed through the steepening of large scale foreshock fluctuations (e.g., Schwartz and Burgess, 1991; Lucek et al., 2008; Omidi et al., 2005; Blanco-Cano et al., 2009). This process can be interpreted to result in an uneven, rippled shock surface. Consequently, Hietala et al. $(2009,2012)$ suggested that HSJs are formed when the solar wind plasma passes through locally inclined parts of such a rippled shock surface, undergoing below average deceleration and thermalization.

In all the studies mentioned above, reconnection could be excluded as a driver for HSJs. In general, however, reconnection can generate exhaust jets near current sheets. These jets are also local. Exhaust jets may be observed close to the magnetopause or within the magnetosheath at thin current sheets, e.g., in regions of strong turbulence behind the quasi-parallel bow shock as described in Retinò et al. (2007). Local, smallscale magnetopause reconnection may also occur as a consequence of HSJs impinging on the magnetopause (Hietala et al., 2012).

The importance of high-speed jets actually stems from their potential interaction with the magnetopause. Localized HSJs with enhanced anti-sunward (i.e., negative geocentric solar ecliptic $x$ direction) velocity in the dayside/subsolar magnetosheath that impinge on the magnetopause increase the pressure on it and push it in, causing locally confined indentations. The amplitudes of these indentations can be remarkably large (on the order of an Earth radius) as observations by Shue et al. (2009) and Amata et al. (2011) have shown. Effects on the magnetopause surface should be particularly strong if the corresponding HSJs are supermagnetosonic in the magnetosheath. In that case, secondary, weak shocks form close to the boundary (Hietala et al., 2009).

Magnetopause indentations are accompanied by local disturbances of the Chapman-Ferraro current system (e.g., Glassmeier and Heppner, 1992) that set up innermagnetospheric compressional waves and/or magnetopause surface waves (e.g., Plaschke et al., 2009; Plaschke and Glassmeier, 2011). Hietala et al. (2012) found that related localized enhancements of ionospheric convection are most likely driven by HSJs.

High-speed jets connect structures of their source regions with the magnetopause. They provide "long-range correlations between processes at the bow shock and at the magnetopause" (Savin et al., 2012), possibly enabling foreshock waves and structures to affect the inner magnetosphere. In summary, high-speed jets are a key element in the coupling of processes upstream of the magnetopause with the magnetosphere-ionosphere system.

In this paper, we focus specifically on HSJs in the subsolar magnetosheath that feature a major velocity component in anti-sunward direction, i.e., that potentially impact the magnetopause and affect the magnetosphere/ionosphere system beneath. Particular emphasis is put on identification of the solar wind conditions favorable for HSJ generation and on the comparison of properties of the solar wind, the HSJs, and the plasma in the surrounding/typical magnetosheath.

In contrast to all the studies mentioned above, which are basically case studies, we present a statistical study of HSJs that complements the recently published work of Archer and Horbury (2013). Archer and Horbury used a semi-automatic detection method and focused on dynamic pressure enhancement characteristics as a function of the relative importance of density versus velocity. Their study was based on Time History of Events and Macroscale Interactions during Substorms (THEMIS) data from one dayside season. We introduce a method for completely algorithmic detection of HSJs and apply it to $4 \mathrm{yr}$ of THEMIS data. The method relies on the comparison of solar wind and magnetosheath data rather than on outlier detection with respect to running averages (see, Archer and Horbury, 2013). Hence, it allows for detection of not only isolated HSJs, but also series of HSJs and longer, continuous intervals of increased magnetosheath dynamic pressure.

\section{Data and HSJ selection}

In this study, we used data from the five THEMIS spacecraft (denoted as THA to THE, Angelopoulos, 2008) obtained for 2008 through 2011 (four years). We also used solar wind data from the NASA OMNI high resolution data set (King and Papitashvili, 2005). Solar wind conditions for a particular THEMIS data sample are given by averages of the OMNI measurements for the preceding five minutes.

We automatically surveyed the THEMIS data for dayside, subsolar magnetosheath intervals: time intervals were pre-selected in which any THEMIS spacecraft was within a $30^{\circ}$ wide, Sun-centered cone with tip at Earth (equivalent to $\pm 2 \mathrm{~h}$ of noon in the ecliptic plane) and between 7 and $18 R_{\mathrm{E}}$ from Earth's center. From these pre-selected intervals, magnetosheath intervals were identified from THEMIS Electrostatic Analyzer (ESA, McFadden et al., 2008) ion density measurements surpassing twice the corresponding solar 
wind ion density. Additionally, we required the energy flux of $1 \mathrm{keV}$ ions to be larger than that of $10 \mathrm{keV}$ ions, ensuring exclusion of inner-magnetospheric measurements (data of ESA energy channels closest to but below 1 and $10 \mathrm{keV}$ were used).

Magnetosheath intervals were only selected for times at which all quantities utilized in this study were available (THEMIS ESA ion velocity, ion density, and temperature moments; ESA ion energy flux and Fluxgate Magnetometer (FGM) (Auster et al., 2008) magnetic field measurements; and OMNI magnetic field, ion velocity, ion density and plasma beta data). Finally, we only kept intervals longer than 2 min. With these criteria, we obtain $2736.9 \mathrm{~h}$ of THEMIS magnetosheath and OMNI solar wind data distributed among 6960 individual intervals (denoted as magnetosheath intervals in this paper).

We selected high-speed jets observed during these intervals that potentially impact the magnetopause, i.e., that feature substantial dynamic pressure in the anti-sunward direction:

$p_{\mathrm{d}, x}=\rho v_{x}^{2}$

where $\rho$ is the ion mass density (assuming protons only) and $v_{x}$ the ion velocity in the geocentric solar ecliptic (GSE) $x$ direction. The following selection criteria (illustrated in Fig. 1) were used:

1. Within a HSJ, the dynamic pressure in GSE $x$ direction $p_{\mathrm{d}, \mathrm{msh}, x}$ shall exceed half the solar wind dynamic pressure $p_{\mathrm{d}, \mathrm{sw}}$ (Fig. 1e: black line surpassing blue line). This criterion prevents selection of HSJs resulting only from solar wind dynamic pressure increases. The time of the maximum pressure ratio between the magnetosheath (MSH) and the solar wind (SW) is denoted by $t_{0}$. The HSJ interval (between the long dashed lines in the figure) is then given by the times around $t_{0}$ where $p_{\mathrm{d}, \mathrm{msh}, x}>0.25 p_{\mathrm{d}, \mathrm{sw}}$ (black line surpassing the green line in Fig. 1e).

2. One-minute-long intervals before and after the HSJ intervals are called pre-HSJ and post-HSJ intervals (delimited by dotted and long dashed lines in the figure), respectively. All three intervals (pre-HSJ, HSJ, and post-HSJ) must lie within a magnetosheath data interval.

3. The ion velocity in GSE $x$ direction $\left(v_{\mathrm{msh}, x}\right)$ shall be negative throughout the HSJ intervals (blue line in Fig. $1 \mathrm{~b}$ between vertical long dashed lines). This ensures that only HSJs directed toward the magnetopause are counted.

4. Within both pre- and post-HSJ intervals, $v_{\mathrm{msh}, x}>$ $v_{\mathrm{msh}, x}\left(t_{0}\right) / 2$ shall be observed (blue line in Fig. 1b above the horizontal dash-dotted line). This criterion

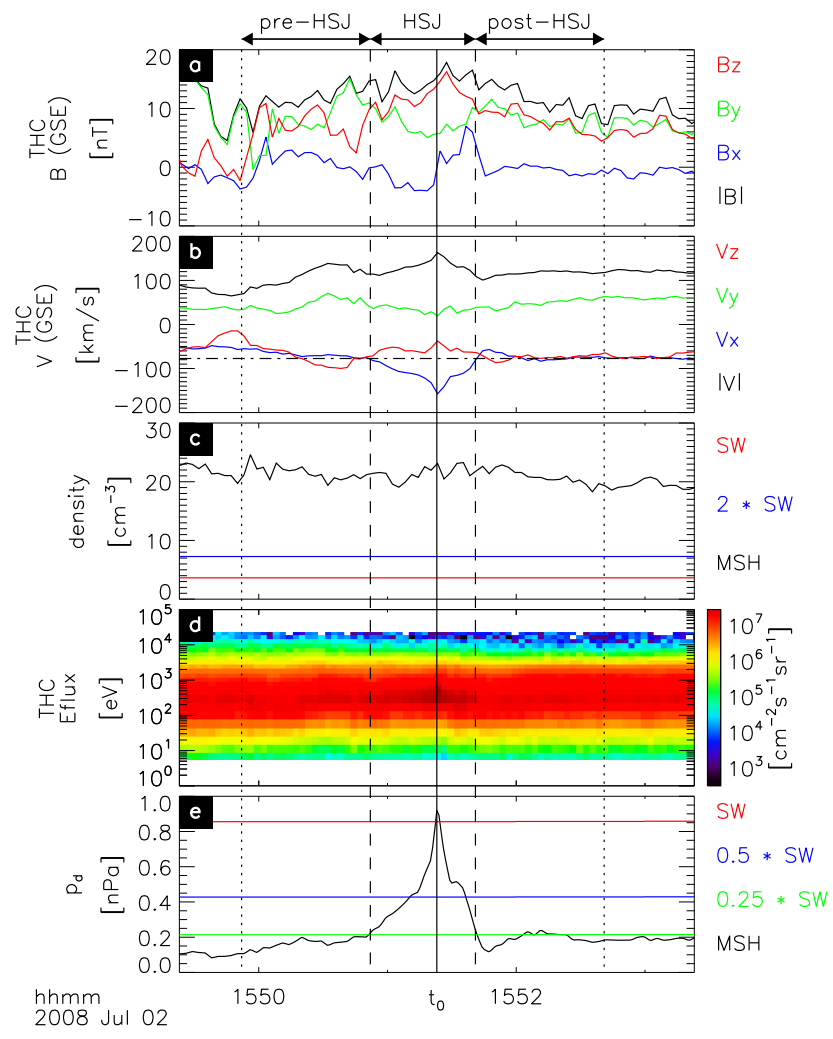

Fig. 1. A high-speed jet observed by THC on 2 July 2008 illustrating the selection criteria. From top to bottom, (panels a to e): magnetic and ion velocity measurements in GSE, ion density, ion energy flux density, and dynamic pressures. (c) shows THC magnetosheath (MSH) measurements in black, and the corresponding OMNI solar wind (SW) ion densities ( $n_{\text {sw }}$ and $\left.2 n_{\mathrm{sw}}\right)$ are shown in red and blue, respectively. (e) depicts GSE $x$ component dynamic pressure $\left(p_{\mathrm{d}, \mathrm{msh}, x}\right)$ at THC in black; corresponding solar wind data $\left(p_{\mathrm{d}, \mathrm{sw}}, p_{\mathrm{d}, \mathrm{sw}} / 2\right.$, and $\left.p_{\mathrm{d}, \mathrm{sw}} / 4\right)$ are shown in red, blue, and green.

ensures that the HSJs are truly jets, featuring antisunward velocity enhancements confined to the respective HSJ intervals, and not simply density enhancements within a steady flow.

These criteria yield a total of 2859 HSJs. In the following sections, we present and discuss statistical results of magnetosheath, HSJ, pre-HSJ, and solar wind data. High-speed jet data belong to samples denoted by $t_{0}$. Pre-HSJ values are median values of those samples from the respective pre-HSJ intervals for which $p_{\mathrm{d}, \mathrm{msh}, x}<0.25 p_{\mathrm{d}, \mathrm{sw}}$ holds. Solar wind conditions for a certain HSJ are given by the average of the available OMNI data samples from the five-minute interval preceding $t_{0}$ (see above).

\section{Results}

The projected locations of the THEMIS spacecraft while in the magnetosheath, according to our selection criteria, are 


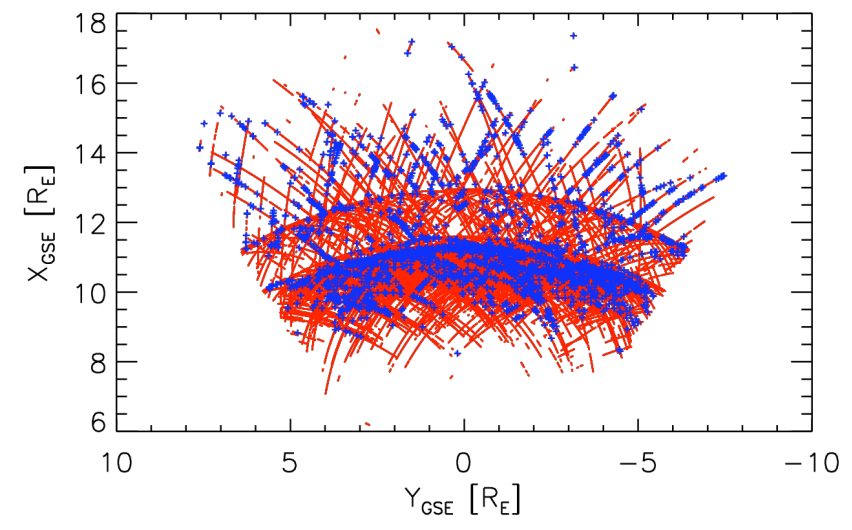

Fig. 2. Coverage of magnetosheath intervals (red), projected onto the GSE $x-y$ plane. THEMIS positions at times $t_{0}$ (HSJs) are marked with blue crosses.

displayed in Fig. 2 in red. Locations at which high-speed jets were observed are depicted by blue crosses. As can be seen, the spatial distribution of the magnetosheath and corresponding HSJ measurements is subject to an orbital bias; most HSJs are observed close to the apogees of the three inner THEMIS spacecraft THA, THD, and THE.

This orbital bias can be more clearly seen in Fig. 3. We calculated the distances of the THEMIS spacecraft to a model magnetopause (by Shue et al., 1998) and a model bow shock (by Merka et al., 2005) along lines radially connecting the center of the Earth with the spacecraft. Figure 3 shows distributions of the relative (i.e., normalized) positions of the spacecraft on these lines with respect to the model magnetopause and bow shock boundaries. The distribution depicted in red pertains to all magnetosheath (MSH) intervals (reference distribution), the blue distribution to the HSJ observations only. OMNI solar wind conditions were used as input parameters for the models.

Due to orbital bias, the reference distribution maximizes closer to the magnetopause than to the bow shock. We find $13 \%$ of the magnetosheath data to be outside the model boundaries, and mainly inside the model magnetopause. The HSJ distribution maximizes closer to the magnetosheath center. Here, $7.1 \%$ of the HSJs are outside the model magnetosheath, with $4.8 \%$ inside the model magnetopause and $2.3 \%$ outside the model bow shock. Normalizing the HSJ by the reference distribution, we obtain the distribution depicted in black in Fig. 3, which clearly indicates that HSJs are much more frequent closer to the bow shock than to the magnetopause.

Temporal and spatial scales of the selected HSJs are shown in Fig. 4. Panel a depicts the distribution of HSJ interval lengths (between long dashed lines in Fig. 1). Although a few tens of seconds can be regarded as typical, HSJs with increased dynamic pressure towards the magnetopause can last up to a few minutes. The distribution maximizes around

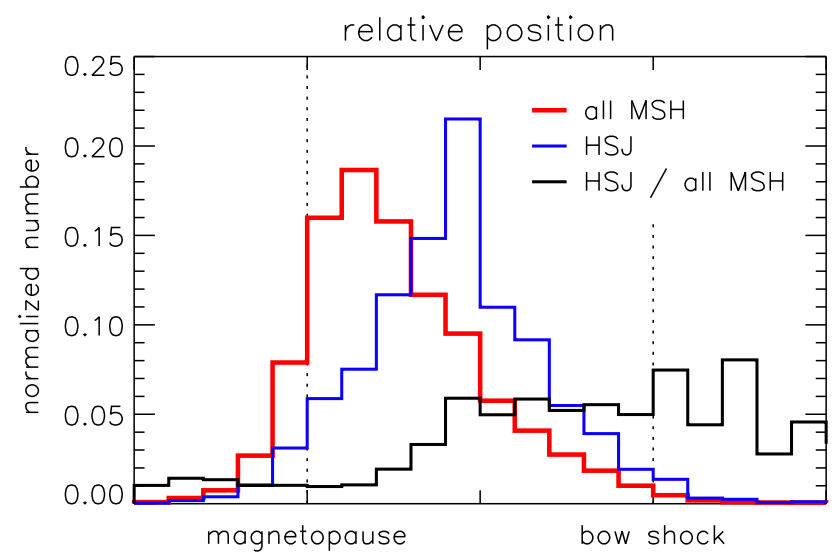

Fig. 3. Distributions of normalized radial distances of all magnetosheath (MSH) observations (red, reference) and HSJ observations (blue) between model magnetopause and bow shock (models by Shue et al., 1998; Merka et al., 2005). The HSJ observation distribution normalized by the reference distribution is shown in black.

a median value of $29 \mathrm{~s}$, in excellent agreement with observations by Savin et al. (2008) and Archer and Horbury (2013).

Integrating the velocity $v_{\mathrm{msh}, x}$ over the HSJ intervals yields the distribution shown in panel $b$. This distribution depicts the range of HSJ spatial lengths in the GSE $x$ direction. The range and median $(\sim 4000 \mathrm{~km})$ of that distribution correspond well with findings by Archer et al. (2012) and Karlsson et al. (2012); the latter reported typical scale sizes of magnetosheath density enhancements in the 0.1 to $10 R_{\mathrm{E}}$ range. As expected, a clear correlation exists between HSJ temporal and spatial length scales (correlation coefficient: 0.91). It should be noted that the temporal and spatial length scales are dependent on the choice of the dynamic pressure ratio threshold level defining the HSJ intervals (we used $p_{\mathrm{d}, \mathrm{msh}, x} / p_{\mathrm{d}, \mathrm{sw}}>0.25$ as selection criterion). In addition, the accuracy of the spatial scale estimates depends on how well the jet structures (dynamic pressure enhancements) follow the plasma bulk flow. If they propagate slower/faster than the bulk velocity, then HSJ spatial scales will be over/underestimated by the integral of $v_{\mathrm{msh}, x}$.

The last panel, c, shows HSJ recurrence time, the distribution of interval lengths between any two subsequent HSJs within a common magnetosheath interval. The distribution covers three orders of magnitude, with inter-HSJ times ranging from 6 to $8765 \mathrm{~s}$ (median: $140 \mathrm{~s}$ ). A distinct time of recurrence is not apparent.

Main properties of the solar wind and magnetosheath plasmas during sheath intervals and pre-HSJ intervals, and at HSJ $t_{0}$ times are shown in Fig. 5. This figure depicts distributions of the GSE $x$ direction dynamic pressure $p_{\mathrm{d}, x}$ and negative ion velocity $-v_{x}$ (top two rows), ion density $n$ (third row), and modulus of the magnetic field $|\boldsymbol{B}|$ (bottom row). The left column presents OMNI solar wind data (red for all 

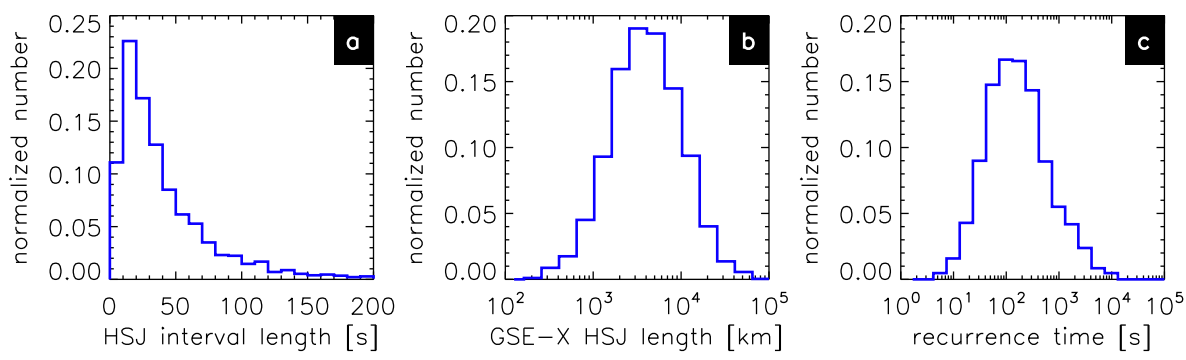

Fig. 4. Scale lengths of HSJs: distributions of (a) temporal lengths of HSJ intervals, (b) spatial lengths in GSE $x$ direction given by the integral of $v_{\mathrm{msh}, x}$ over the HSJ interval, and (c) recurrence time, i.e., time between any two subsequent HSJs within a common magnetosheath interval.

magnetosheath intervals, blue for HSJ $t_{0}$ times). The middle column is based on THEMIS magnetosheath data (blue: HSJ $t_{0}$ times, green: corresponding pre-HSJ interval data). In the right column, ratios of solar wind to magnetosheath data are shown (red: all sheath intervals, blue: HSJ $t_{0}$ times, green: pre-HSJ intervals). Black dotted lines depict the distributions of ratios of pre-HSJ to HSJ $t_{0}$ values. The median values of the ratio distributions are shown in Table 1.

As can be seen in the left column of Fig. 5, distributions of solar wind data belonging to HSJ times (in blue) are similar to the reference distributions in red. Dynamic pressures seem to be slightly enhanced during HSJ times (panel a). The median values of the dynamic pressure distributions are $1.54 \mathrm{nPa}$ (reference) and $1.82 \mathrm{nPa}$ (HSJs). The dynamic pressure is a combined quantity of velocity and density: comparison of HSJ and reference distributions in panels $\mathrm{d}$ and $\mathrm{g}$ of Fig. 5 yields that HSJ occurrence is enhanced for higher solar wind velocities $v_{\mathrm{sw}}$ and lower densities $n_{\mathrm{sw}}$. The shift in HSJ $n_{\text {sw }}$ distribution indicates that increased dynamic pressure is not important in HSJ generation. Otherwise, higher solar wind densities would also be associated with increased HSJ occurrence. Medians of reference (and HSJ) distributions of ion velocity and density in the solar wind are $357 \mathrm{~km} \mathrm{~s}^{-1}$ $\left(400 \mathrm{~km} \mathrm{~s}^{-1}\right)$ and $6.81 \mathrm{~cm}^{-3}\left(5.84 \mathrm{~cm}^{-3}\right)$, respectively. Distributions of $\left|\boldsymbol{B}_{\mathrm{sw}}\right|$ shown in panel $\mathrm{j}$ are extremely similar. Hence, HSJ occurrence does not appear to be dependent on that quantity.

As shown in the middle column of Fig. 5, the differences between magnetosheath HSJ plasma parameters and plasma conditions outside the jets (pre-HSJ intervals) can be much larger. Panel e shows the pre-HSJ and HSJ $t_{0}$ distributions of the anti-sunward ion velocity $\left(-v_{\mathrm{msh}, x}\right)$. Velocities under $100 \mathrm{~km} \mathrm{~s}^{-1}$ are typical in the subsolar sheath. Within HSJs, however, much higher velocities (mostly above $100 \mathrm{~km} \mathrm{~s}^{-1}$, and up to several hundred $\mathrm{km} \mathrm{s}^{-1}$ ) are observed. This result is related to the HSJ selection criterion that requires high dynamic pressure ratios $p_{\mathrm{d}, \mathrm{msh}, x} / p_{\mathrm{d}, \mathrm{sw}}$. Consequently, distributions of HSJ and pre-HSJ magnetosheath dynamic pressures (panel b) differ by almost an order of magnitude.

Further analysis of the pre-HSJ and HSJ velocity vectors indicates that the HSJs are deflected towards the Earth-Sun
Table 1. Median values of ratio distributions shown in Fig. 5 (panels c, f, i, and 1 ).

\begin{tabular}{lrrrr}
\hline & $p_{\mathrm{d}, x}$ & $-v_{x}$ & $n$ & $|\boldsymbol{B}|$ \\
\hline SW / MSH & 18.1 & 8.36 & 0.24 & 0.18 \\
SW / HSJ & 1.23 & 2.71 & 0.16 & 0.22 \\
SW / pre-HSJ & 7.17 & 5.86 & 0.22 & 0.24 \\
pre-HSJ / HSJ & 0.15 & 0.46 & 0.74 & 0.91 \\
\hline
\end{tabular}

line in comparison to the pre-HSJ data (not shown). The angles between velocity vectors and the GSE $x$ direction are only about half as large for HSJs as for pre-HSJ intervals. The median of the angle ratio distribution is 0.57 . Although such deflections are in agreement with observations by Karlsson et al. (2012) and Hietala et al. (2012), in this study they also must be attributed to HSJ selection. The median deflection angle between pre-HSJ and HSJ velocities is $28.6^{\circ}$.

As depicted in Fig. 5h and k, slightly higher densities and magnetic fields are also found within the HSJs than in preHSJ values, but these changes are not as significant as the changes in velocity. Thus, the selected HSJs are truly jets and not simply density enhancements within an otherwise unaltered magnetosheath plasma flow.

The ratios of solar wind/magnetosheath dynamic pressures (Fig. 5c) are also closely related to HSJ and pre-HSJ selection criteria. High-speed jets are required to have pressure ratios of less than 2; pre-HSJ values are taken only from samples for which pressure ratios over 4 are observed. These criteria explain the sharp cut-off values of the blue and green distributions in Fig. 5c. The close relationship between dynamic pressure and velocity behavior is also reflected in the ratio distributions of both quantities (the similarity between panels $\mathrm{c}$ and $\mathrm{f}$ of Fig. 5).

Similarities between panels $i$ and 1 of that figure are also apparent. The distributions of pre-HSJ to HSJ values (dotted lines) maximize close to but below 1 , in agreement with higher densities and magnetic fields within the HSJs than within the surrounding plasma (on average). Higher densities are observed in $89.5 \%$ of the 2859 HSJs. Hence, our criteria favor the selection of these higher density exhibiting HSJs 

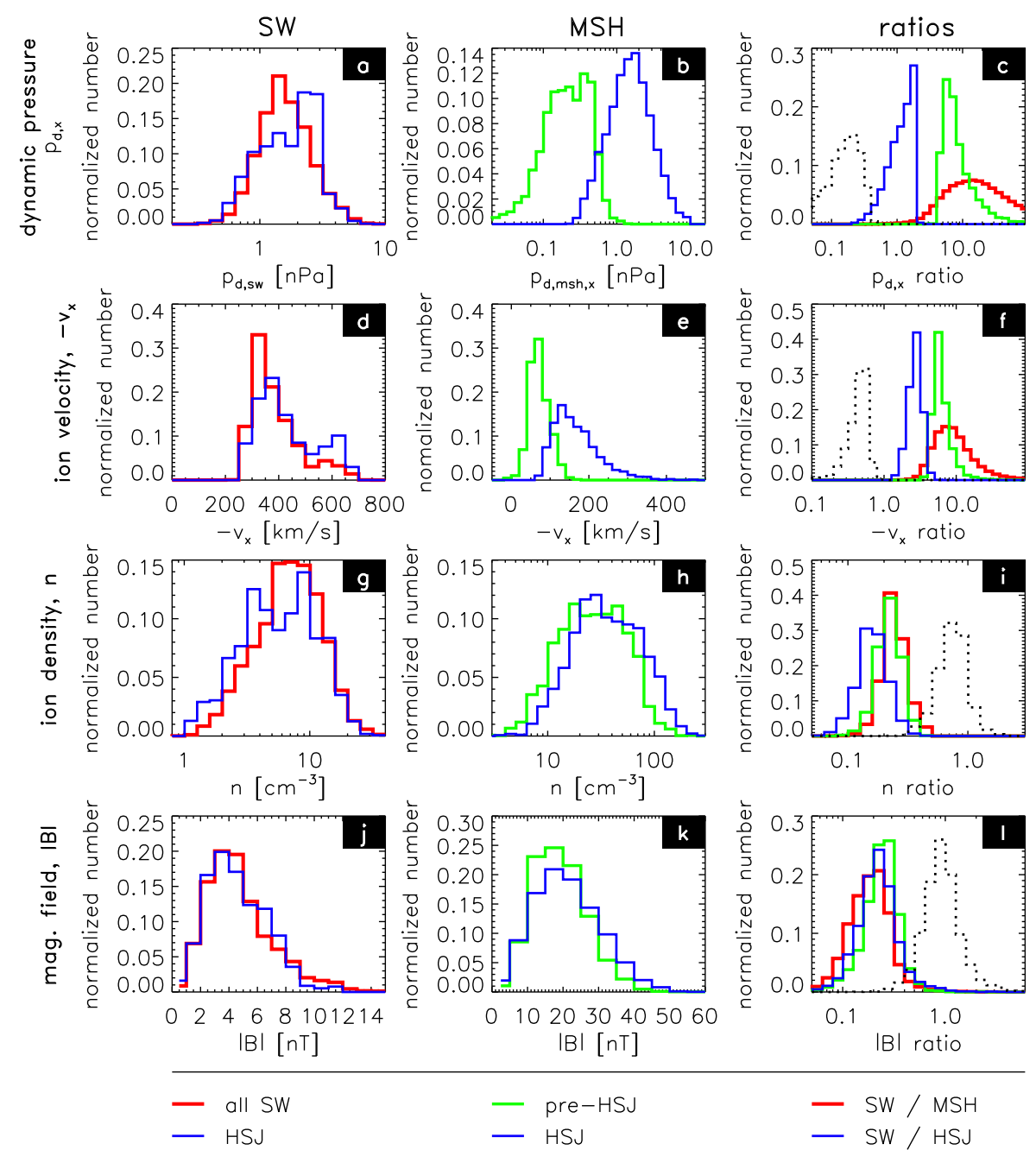

- pre-HSJ
$-\quad$ HSJ

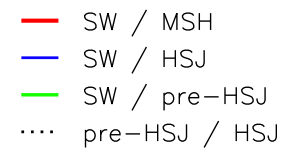

Fig. 5. Distributions of solar wind (SW) and magnetosheath (MSH) data for all sheath intervals, HSJs, and pre-HSJ times, as well as ratio distributions. Rows (top to bottom) correspond to GSE $x$ direction dynamic pressure $\left(p_{\mathrm{d}, \mathrm{x}}\right)$ and ion velocity $\left(-v_{x}\right)$, ion density $(n)$, and modulus of the magnetic field $(|\boldsymbol{B}|)$ measurements. Left column: distributions of OMNI solar wind data corresponding to HSJ $t_{0}$ times (blue) as well as to all times for which magnetosheath measurements are available (red). Middle column: distributions of THEMIS sheath data corresponding to HSJ $t_{0}$ times (blue) as well as to pre-HSJ times (green). Right column: ratios of OMNI and THEMIS data: for all selected sheath intervals (red); for HSJ $t_{0}$ times (blue); and for pre-HSJ intervals (green). Ratios of pre-HSJ to HSJ data are depicted by dotted black lines.

even more than does the method used by Archer and Horbury (2013), who found that only $82 \%$ of their pressure enhancements featured higher densities. Increases and decreases in HSJ magnetic field are more balanced: though a majority of HSJs (61\%) feature a magnetic field increase with respect to the surrounding plasma, a significant fraction (39\%) exhibit a field reduction.

Increases in density and velocity within HSJs agree with most previous observations (e.g., Archer et al., 2012; Amata et al., 2011; Savin et al., 2008). In our study, the increase in velocity clearly dominates the behavior in dynamic pressure, justifying the designation of the dynamic pressure enhancements as jets. This contrasts, for instance, with results by Savin et al. (2008) who found kinetic energy density enhancements to be usually density dominated, thus corresponding more to "plasma compressions moving at enhanced magnetosheath speeds".

Distributions of Alfvén and magnetosonic Mach numbers are depicted in Fig. 6. Only GSE $x$ component velocity measurements were used in the computations. Hence, Mach numbers correspond only to the plasma velocity in the anti-sunward direction, toward the magnetopause in the subsolar magnetosheath. 

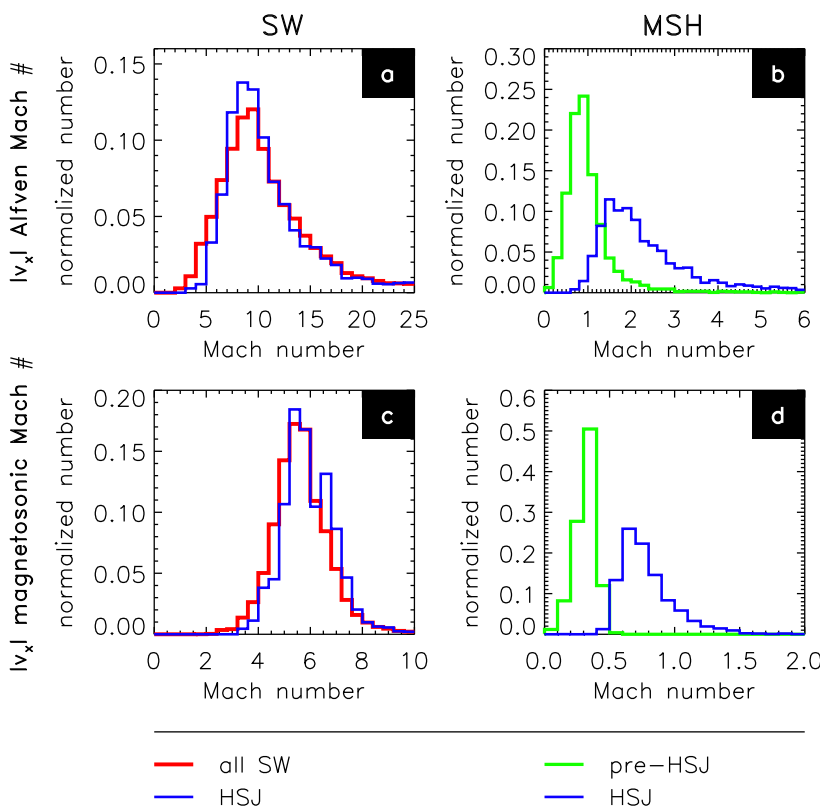

Fig. 6. Distributions of solar wind (SW) and magnetosheath (MSH) data for all sheath intervals, HSJs, and pre-HSJ times: rows (top and bottom) correspond to Alfvén and magnetosonic Mach numbers computed from GSE $x$ direction ion velocity moments only. Left column: distributions of OMNI solar wind data corresponding to all times for which magnetosheath measurements are available (red), as well as solar wind data corresponding to HSJ $t_{0}$ times only (blue). Right column: distributions of THEMIS sheath data corresponding to HSJ $t_{0}$ times (blue) as well as to pre-HSJ intervals (green).

Panels a and $\mathrm{c}$ show that the HSJ distributions (blue) are quite similar to the reference solar wind distributions (red). This holds in particular for the Alfvén Mach number distributions. The median Alfvén and magnetosonic Mach numbers of the distributions depicted in these panels are: 10.0 and 5.6 (solar wind reference), as well as 10.0 and 5.9 (solar wind at HSJ $t_{0}$ times). Large differences between HSJ and preHSJ interval data are observed in the magnetosheath (panels $b$ and d): Mach numbers within the HSJs are significantly enhanced. In the GSE $x$ direction, pre-HSJ magnetosonic Mach numbers are always below 1, whereas $14.2 \%$ of the 2859 HSJs exhibit super-magnetosonic anti-sunward flows. Super-Alfvénic speeds in the GSE $x$ direction are seen during $36.6 \%$ of the pre-HSJ intervals. At HSJ $t_{0}$ times, this number increases to $98.1 \%$. Hence, almost all HSJs are superAlfvénic in the anti-sunward direction alone.

Figure 7 addresses the effect of solar wind variability on HSJ occurrence. As stated above, solar wind parameters corresponding to HSJ $t_{0}$ times are averages of OMNI measurements from the 5 min preceding $t_{0}$. We also computed the distribution of maximum angular deviations between any two IMF entries in the OMNI solar wind data set within these 5min intervals (if the OMNI data were available without data gaps). This distribution is depicted in blue in Fig. 7a. The
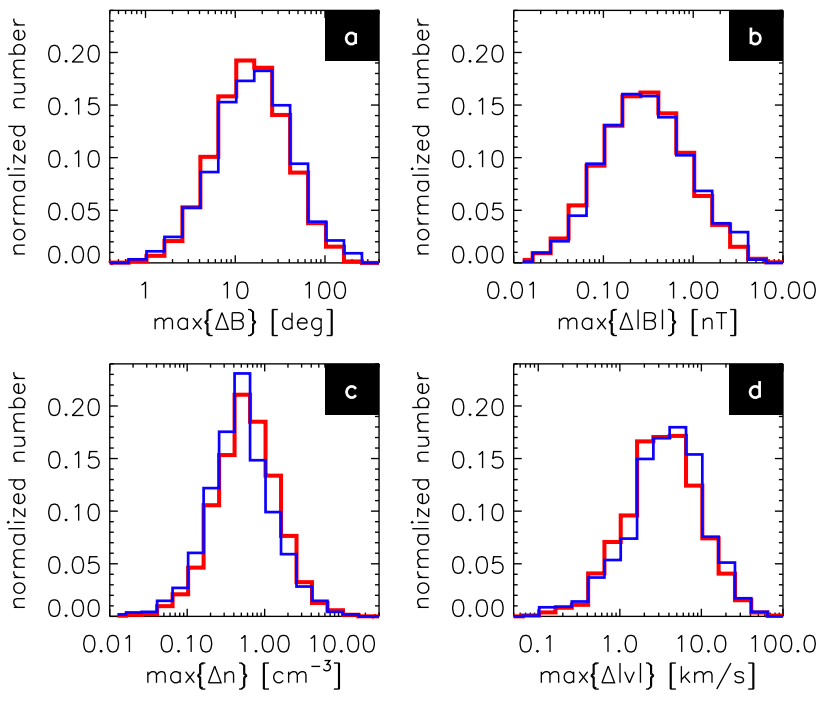

- all SW

- HSJ

Fig. 7. Distributions of maximum variations in (a) the angle of the IMF, (b) the strength of the IMF, (c) the solar wind (SW) density, and (d) the solar wind velocity for all magnetosheath intervals (red) and for intervals preceding HSJ $t_{0}$ times (blue).

median value is only $16^{\circ}$, i.e., the IMF changed less than $16^{\circ}$ in direction before half of the HSJs. Hence, large deflections in IMF do not seem to be associated with a majority of HSJs (see also Archer and Horbury, 2013). The distribution depicted in red in the same panel shows the angular variability for all samples of the magnetosheath intervals. This distribution is a reference distribution of IMF variability. The red and blue distributions almost coincide, allowing us to further infer that IMF variability does not control the occurrence of a significant fraction of HSJs.

The other three panels of Fig. 7 show general solar wind (red) and HSJ (blue) distributions of the variability of IMF strength $\left|\boldsymbol{B}_{\mathrm{sw}}\right|$ (panel b), solar wind density $n_{\mathrm{sw}}$ (panel c), and solar wind velocity $\left|\boldsymbol{v}_{\mathrm{sw}}\right|$ (panel d). These distributions were computed in a manner similar to that for the distributions shown in panel a. As can be seen, red and blue distributions agree very well in each of the panels. Hence, variability in the respective quantities does not seem to (strongly) control HSJ occurrence in the magnetosheath, either. At best, a slight preference for lower/higher variability in solar wind density/velocity is perceptible (panels $\mathrm{c}$ and $\mathrm{d}$ ).

Our results show that neither the solar wind Mach numbers nor the density, velocity, and magnetic field strength, nor the variability in these solar wind quantities seem to be strongly controlling the occurrence of HSJs in the subsolar magnetosheath. The only parameter with a major influence on their appearance is the IMF cone angle, i.e., the angle of the IMF to the Earth-Sun line. Distributions of this quantity are depicted in Fig. 8; when comparing a reference distribution of cone angles (shown in red) with the distribution 


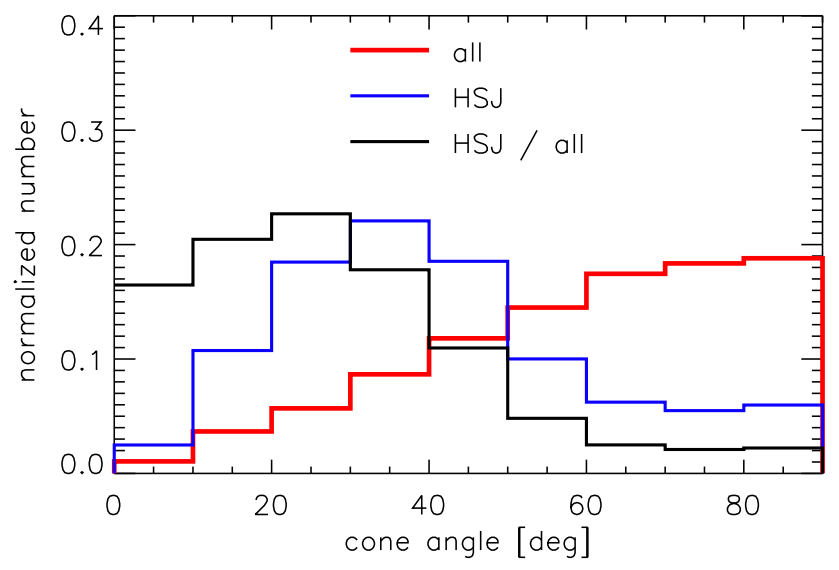

Fig. 8. Distributions of IMF cone angles for HSJ $t_{0}$ times (blue) as well as for intervals of magnetosheath measurement availability (red). The ratio of the former and latter distributions (normalized HSJ IMF cone angle distribution) is shown in black.

at HSJ $t_{0}$ times (in blue), we find that HSJs in the subsolar magnetosheath generally correspond to lower cone angles $\left(<45^{\circ}\right.$ : 1842 out of 2859 HSJs, $\left.64.4 \%\right)$. Radial IMF is a favorable solar wind condition in agreement with, e.g., Hietala et al. (2009), Hietala et al. (2012), and Archer and Horbury (2013). In particular, the normalized distribution of HSJ cone angles (black) shows a clear tendency of HSJs to occur at lower $\left(<45^{\circ}\right)$ cone angles. Hence, high-speed jets occur much more often behind the quasi-parallel bow shock, which suggests a causal relationship of HSJ occurrence to the presence of the quasi-parallel bow shock or the foreshock region upstream.

The last two Figs., 9 and 10, show a comparison between the temperature and pressure characteristics of HSJs and the surrounding plasma in the magnetosheath. Panel a of Fig. 9 depicts distributions of the quotients of the magnetosheath ion temperatures perpendicular and parallel to the magnetic field $\left(T_{\text {perp }} / T_{\text {para }}\right)$, in general (red, reference distribution), during pre-HSJ intervals (green), and at HSJ $t_{0}$ times (blue). We can see that $T_{\text {perp }}$ is usually enhanced over $T_{\text {para }}$. In and around HSJs, however, both temperatures are more similar (isotropic), as the corresponding distributions maximize around 1 (see also observations by Savin et al., 2008; Amata et al., 2011; Archer et al., 2012).

The second panel, b, of Fig. 9 shows the distributions of pre-HSJ to HSJ $t_{0}$ temperature ratios. They are shifted toward values higher than 1 . Hence, temperatures within HSJs are typically diminished with respect to the magnetosheath plasma surrounding them (Savin et al., 2008; Archer et al., 2012). The difference is larger for $T_{\text {perp }}$, accounting for the temperature being even more isotropic within HSJs than outside. Higher $T_{\text {perp }}$ and $T_{\text {para }}$ within HSJs (ratios below 1) are observed for $12.2 \%$ and $19.3 \%$ of the 2859 HSJs, respectively.
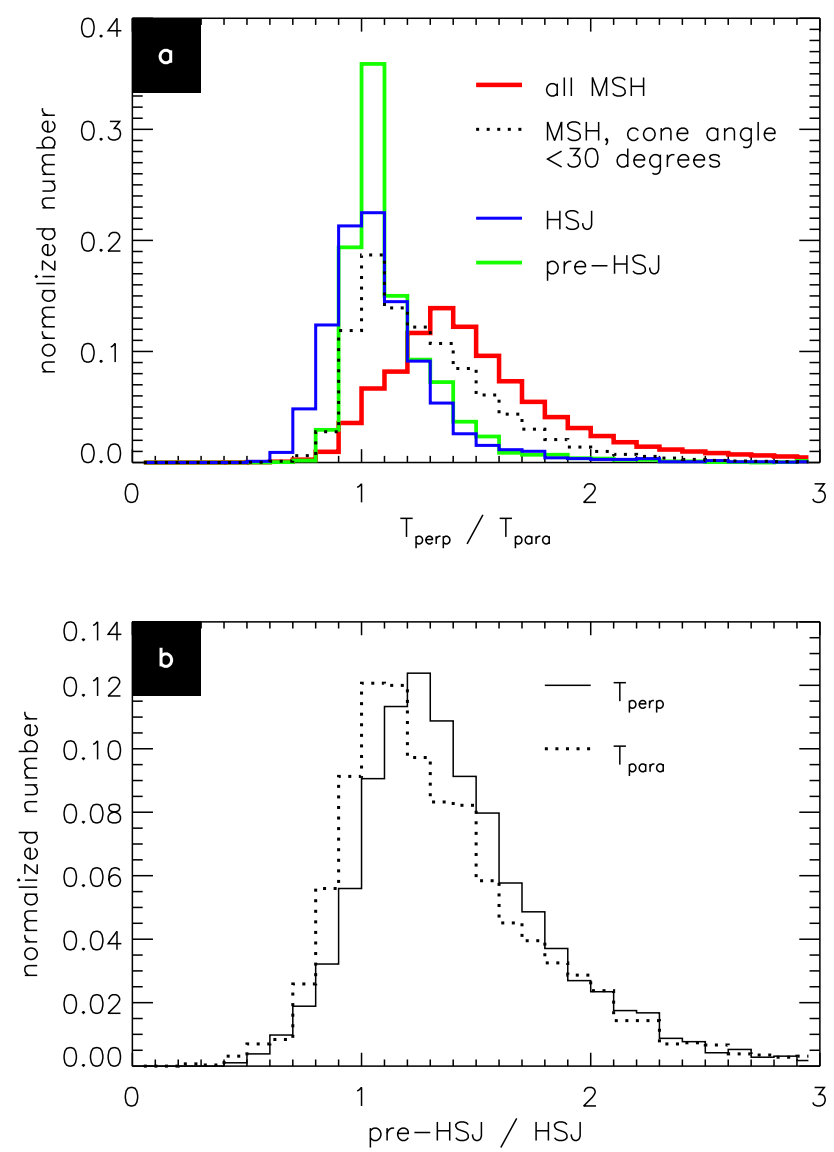

Fig. 9. Upper panel (a): distributions of perpendicular over parallel ion temperatures ( $\left.T_{\text {perp }} / T_{\text {para }}\right)$ of all selected magnetosheath (MSH) times (red), magnetosheath samples measured during low IMF cone angle conditions $\left(<30^{\circ}\right.$; dotted black), pre-HSJ intervals (green), and HSJ $t_{0}$ times (blue). Lower panel (b): pre-HSJ over HSJ distributions of $T_{\text {perp }}$ (solid line) and $T_{\text {para }}$ (dotted line).

Finally, Fig. 10 shows pressure ratios between pre-HSJ and HSJ magnetosheath measurements. Ratio distributions of the dynamic pressure in the GSE $x$ direction ( $p_{\mathrm{d}, \mathrm{msh}, x}$; dotted line), the magnetic pressure ( $p_{\mathrm{mag}, \mathrm{msh}}$; dashed line), the thermal pressure ( $p_{\mathrm{th}, \mathrm{msh}}$; dash-dotted line), and the sum of these pressures ( $p_{\text {tot, msh, } x}$; solid line) are depicted. We can see that the total pressure distribution maximizes at around 0.5 . Hence, the pressure of the HSJ plasma acting toward the magnetopause is about twice as large as in the surrounding plasma. This increase in pressure is based on the large difference in dynamic pressure, the median of the corresponding ratio distribution (dotted line) being 0.15 . Hence, preHSJ and HSJ $p_{\mathrm{d}, \mathrm{msh}, x}$ typically differ by a factor close to 6.5. The magnetic pressure $p_{\mathrm{mag}, \mathrm{msh}}$ increases slightly inside the HSJs (median pre-HSJ to HSJ ratio: 0.83), in agreement with results shown in Fig. 5k. The median ratio of pre-HSJ to HSJ thermal pressures ( $\left.p_{\text {th,msh }}\right)$ is 0.86 , comparable to the result for $p_{\text {mag,msh }}$, although the distribution of $p_{\text {th,msh }}$ ratios is much more concentrated around that value. Increases 


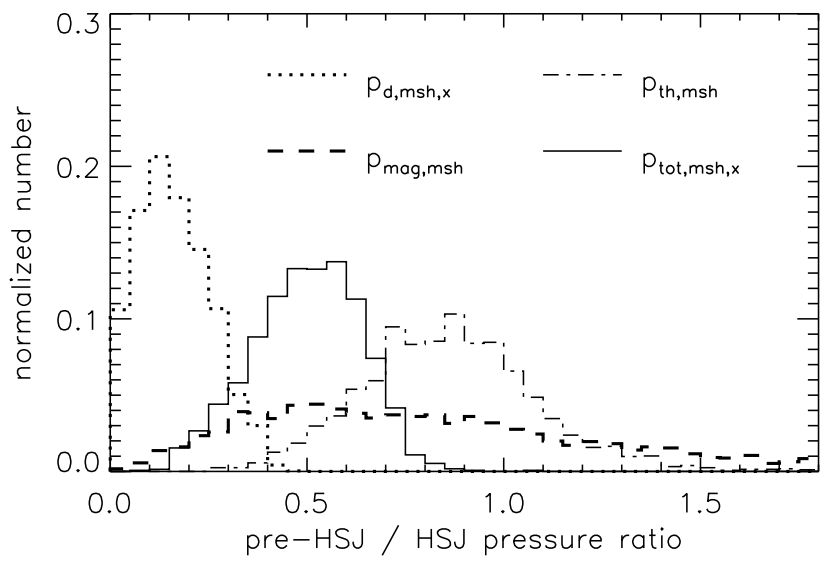

Fig. 10. Distributions of pre-HSJ over HSJ magnetosheath pressure ratios of the dynamic pressure in the GSE $x$ direction $\left(p_{\mathrm{d}, \mathrm{msh}, x}\right.$;

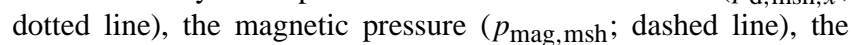
thermal pressure $\left(p_{\text {th,msh }}\right.$; dash-dotted line), and the sum of the above pressures ( $p_{\text {tot, msh, } x}$; solid line).

in density within the HSJs (Fig. 5h) are partially offset by decreases in temperature, as shown in Fig. 9b.

\section{Discussion}

As shown in the previous section, increased levels of solar wind variability do not seem to be associated with the majority of HSJs (see Fig. 7). As a result, the interaction of prominent rotational discontinuities with the bow shock (see Lin et al., 1996a, b) or the existence of hot flow anomalies (Savin et al., 2012) cannot be responsible for the typical HSJs in our database. However, our criteria for HSJ selection are also expected to exclude jets generated by interaction of rotational discontinuities with the bow shock, as they produce dynamic pressure pulses with enhancements in the boundary tangential velocity components perpendicular to $v_{x}$ in the subsolar magnetosheath (Lin et al., 1996a, b).

Furthermore, almost all HSJs are super-Alfvénic, even when only taking into account $v_{\mathrm{msh}, x}$ (see Fig. 6b). This result suggests that reconnection can also be excluded as major driving mechanism of HSJs. Otherwise, typical reconnection outflow jet speeds on the order of the Alfvén velocity (less, if only taking into account the velocity component in GSE $x$ direction) would be expected (Priest and Forbes, 2000). In any case, the selection criteria would only allow for reconnection within the magnetosheath (e.g., at thin current sheets, Retinò et al., 2007) to contribute to the HSJ database, as reconnection jets at the subsolar magnetopause would have major boundary tangential velocity components and not a major component in the GSE $x$ direction. The scale sizes of thin current sheet reconnection jets $(\sim 1 \mathrm{~s}$ and $\sim 100 \mathrm{~km}$, Retinò et al., 2007), however, seem to be at least one order of magnitude too small compared with the typical HSJs in our data set (see Fig. 4).

The only solar wind parameter we find to control HSJ occurrence is the IMF cone angle (Fig. 8). Low IMF cone angles clearly favor HSJ generation in the subsolar magnetosheath. Hence, HSJs are far more often observed behind the quasi-parallel bow shock, in agreement with results from, e.g., Němeček et al. (1998), Hietala et al. (2009, 2012), and Archer and Horbury (2013). This result is consistent with HSJ generation via bow shock ripples (Hietala et al., 2009), as enhanced instability and major undulations are expected features of the quasi-parallel bow shock (e.g., Burgess et al., 2005). Large scale undulations (ripples) enable the passage of less thermalized, high-speed solar wind plasma into the magnetosheath at locations of higher bow shock inclination. Furthermore, as shown in the previous section, HSJ occurrence is enhanced in the outer magnetosheath, closer to the bow shock than to the magnetopause (Fig. 3). Hence, we can infer that HSJ generation should take place close to the bow shock, at the bow shock, or in the foreshock region upstream.

As can be seen in Fig. 9a, ion temperatures in the HSJs and surrounding plasma are much more isotropic than usual in the magnetosheath ( $T_{\text {perp }} / T_{\text {para }}$ close to 1$)$. In general, $T_{\text {perp }}$ dominates $T_{\text {para }}$ in the sheath, as indicated by the red line in that panel (e.g., Crooker et al., 1976; Czaykowska et al., 2001). However, behind the quasi-parallel bow shock, temperatures are also known to be more isotropic (e.g., Ellacott and Wilkinson, 2007). This is also confirmed by our set of sheath measurements: samples pertaining to low IMF cone angle conditions $\left(<30^{\circ}\right)$ yield the distribution depicted by a dotted black line in Fig. 9a. This distribution is more similar to the pre-HSJ distribution (green line) than to the reference distribution of all magnetosheath samples (red line). Hence, the difference between pre-HSJ or HSJ and general magnetosheath temperature ratio distributions, and the similarity between pre-HSJ and HSJ distributions can at least partly be explained by most HSJs being observed behind the quasi-parallel bow shock. The same applies to the small differences between reference and HSJ distributions of solar wind velocity, density, and magnetosonic Mach number, shown in Figs. 5d, 5g, and 6c. When using only low IMF cone angle interval data, the reference distributions basically coincide with the HSJ distributions (not shown).

Moreover, the fact that the plasma within the HSJs has lower temperatures (Fig. 9b) than pre-HSJ plasma is also consistent with the bow shock ripple jet generation mechanism. Plasma passing through the locally inclined and, thus, weaker part of a shock (ripple) should be less thermalized. Observations of lower, more isotropic temperatures within HSJs agree with a series of previous studies, e.g., by Savin et al. (2008), Amata et al. (2011), Hietala et al. (2012), and Archer et al. (2012).

Our results, however, do not constitute hard evidence for high-speed jet generation via bow shock ripples. Other mechanisms related to the dayside, quasi-parallel bow shock or the 
foreshock region upstream, present under stable low cone angle IMF conditions, may be equally consistent with our observations.

There are also indications that not all HSJs could have been generated by bow shock ripples. A non-negligible fraction of HSJs actually feature higher temperatures than the surrounding magnetosheath plasma. Furthermore, not all HSJs were observed during low IMF cone angle intervals. A significant fraction of the HSJs (35.6\%) were observed under higher IMF cone angle conditions $\left(>45^{\circ}\right)$.

Interestingly, long intervals of low IMF cone angle conditions do not guarantee observations of HSJs by individual spacecraft present in the subsolar magnetosheath. Out of all the sheath data obtained by the five THEMIS spacecraft during the $4 \mathrm{yr}$ surveyed, we identified 22 intervals over $1 \mathrm{~h}$ in duration in which the IMF cone angle (5-min OMNI data averages, see above) stayed below $30^{\circ}$. Some of these time intervals are counted multiple times because more than one THEMIS spacecraft was in the subsolar magnetosheath (THA: 6 intervals, THB: 2 intervals, THC: 4 intervals, THD and THE: 5 intervals each). The 5 intervals pertaining to THD and the 5 intervals pertaining to THE overlap in time; 4 of those also (partially) coincide with 4 THA intervals. The start times of these 4 time intervals and the counts of HSJ observations by the three spacecraft are listed in Table 2.

Let us now take a closer look at these time intervals. During intervals 1, 3, and 4 (numbers according to the first column in Table 2), either THD and THE observed HSJs while THA did not, or THA observed HSJs while THD and THE did not. Hence, while one spacecraft may not observe HSJs, other spacecraft present in the subsolar sheath at the same time but at a different location might do so. We can conclude that the localized nature of the HSJs may prevent individual spacecraft from registering them even during longer intervals under conditions that are very favorable for HSJ occurrence. Consequently, Fig. $4 c$ depicts the distribution of inter-HSJ times as observed by individual spacecraft at a given position. It does not show the occurrence rate of HSJs everywhere in the magnetosheath.

In our study, we focus on HSJs that potentially impact the magnetopause and affect the magnetosphere/ionosphere system beneath. Hence, the high dynamic pressure in the GSE $x$ direction (compared to solar wind conditions) is a major criterion for our selection of HSJs in the subsolar magnetosheath. The total pressure in that direction accounts for the standoff distance of the magnetopause from Earth. Dynamic pressure of the solar wind is converted into thermal and magnetic pressures at the bow shock and within the magnetosheath. Those act against the magnetospheric magnetic field at the magnetopause, across which pressure balance is maintained. As shown in Fig. 10, the relative importance of the different partial pressures changes dramatically within the HSJs with respect to the sheath plasma outside. Thermal and magnetic pressures are somewhat increased (on average) within HSJs (pre-HSJ to HSJ median ratios of around 0.85).
Table 2. Interval start times corresponding to magnetosheath data intervals of THA, THD, and THE with durations over one hour in which the IMF cone angle stayed below $30^{\circ}$. Counts of HSJ observations by the respective spacecraft during these intervals are listed in the right three columns.

\begin{tabular}{llrrr}
\hline interval \# & start times & THA & THD & THE \\
\hline 1 & $2009-11-0418: 23$ & 2 & 0 & 0 \\
2 & $2010-10-1316: 31$ & 8 & 10 & 10 \\
3 & $2010-11-0616: 49$ & 0 & 4 & 3 \\
4 & $2010-11-0717: 25$ & 0 & 4 & 8 \\
\hline
\end{tabular}

The dynamic pressure, however, increases dramatically (median ratio of 0.15 ), leading to a doubling of total pressure in the GSE $x$ direction.

These large increases in pressure within HSJ are expected to cause large indentations of the magnetopause on impact, as observed by Shue et al. (2009), Amata et al. (2011), and Hietala et al. (2012). Localization of the jets leads to local perturbations, not to global motion of the magnetopause boundary. The effect of boundary deformation is enhanced for stronger HSJs, which may even feature super-magnetosonic speeds in the GSE $x$ direction as shown in Fig. 6d (see also Savin et al., 2012; Hietala et al., 2009). Jet plasma moving toward the magnetopause at supermagnetosonic speeds must be decelerated ahead of the magnetopause, implying the presence of a shock for the HSJ plasma (weak secondary shock, see Hietala et al., 2009).

In concluding this section, we discuss some of the further consequences that may arise from the high-speed jets' interaction with the magnetopause: localized magnetopause indentations imply local disturbances of the Chapman-Ferraro current, as described in Glassmeier and Heppner (1992). These disturbance currents may either be closed via fieldperpendicular currents within the magnetosphere, i.e., generation of compressional waves, or via field-aligned currents at the magnetopause. Plaschke and Glassmeier (2011) showed that magnetopause surface waves may be generated by impinging HSJs. In particular, standing magnetopause surface waves (or Kruskal-Schwarzschild modes) may be created, as local disturbances of the Chapman-Ferraro current and corresponding field-aligned closing currents constitute part of the surface wave current system.

Furthermore, ionospheric effects of HSJs were reported, e.g., by Hietala et al. (2012), who identified enhanced localized and short-lived convection flow channels in the dayside polar ionosphere during an interval of HSJ occurrence using Super Dual Auroral Radar Network (SuperDARN) radar and ground magnetometer measurements. The ionospheric flow enhancements had several properties in common with ionospheric traveling convection vortices and the related magnetic impulse events (Kataoka et al., 2001, 2003). 
In general, the consequences of HSJs can be manifold and are far from being fully understood. Hence, further assessment of the geoeffectiveness of magnetosheath high-speed jets in a comprehensive manner is highly desirable.

\section{Summary and conclusions}

We present a statistical study of high-speed jets in the magnetosheath; this is the first study to focus specifically on jets in the subsolar sheath, which feature a major GSE $x$ component in dynamic pressure with respect to solar wind conditions. Hence, the selected HSJs are directed toward and potentially impact the magnetopause. Furthermore, they do not result from solar wind dynamic pressure increases. The aim of the study is to determine which conditions are favorable for geoeffective HSJ formation and the properties of these jets in the magnetosheath. These are our main findings:

1. High-speed jet occurrence is controlled by IMF cone angle. Radial IMF (low cone angles) favors the generation of HSJs in the subsolar magnetosheath. Furthermore, jets are observed relatively more often closer to the bow shock than to the magnetopause. Hence, we conclude that most of the selected jets are related to the presence of the quasi-parallel bow shock or the foreshock region upstream, consistent with the bow shock ripple based generation mechanism (Hietala et al., 2009). However, our results do not constitute direct evidence in favor of this mechanism; clearly further studies are needed. An analysis comparing the statistical data set introduced here with MHD model calculations of a rippled bow shock is presented in the follow-up study by Hietala and Plaschke (2013).

2. There are also minor dependencies of HSJ occurrence on other solar wind quantities: HSJs tend to be associated with slightly higher solar wind velocities, slightly enhanced magnetosonic Mach numbers, and slightly lower densities, though these trends are themselves related to low IMF cone angle conditions.

3. We found variations in solar wind parameters to have very little influence on HSJ occurrence. This result contrasts with numerous previous studies that point to IMF variations (rotational discontinuities, hot flow anomalies) as causes for HSJ formation (e.g., Lin et al., 1996a, b; Archer et al., 2012; Savin et al., 2012). Fluctuation levels in IMF direction and strength, solar wind density, and velocity before HSJ $t_{0}$ times are almost unchanged in comparison with reference levels.

4. Within the high-speed jets, temperatures are generally found to be lower and more isotropic than in the surrounding or typical magnetosheath plasmas. This is also consistent with bow shock ripples being responsible for HSJ formation, and agrees with previous observations (e.g., Savin et al., 2008; Archer et al., 2012).

5. Additional characteristics of the HSJs are largely increased velocity in the GSE $x$ direction, corresponding dynamic pressure, and Mach numbers (in part due to selection criteria), as well as increased density and magnetic field strength.

6. HSJs are almost always super-Alfvénic (98\%), even though only their velocity component in the antisunward direction is taken into account. They are also partially super-magnetosonic (14\%), implying the presence of a second shock in the magnetosheath, closer to the magnetopause.

7. We find HSJs to be localized: temporal scales of a few tens of seconds (median: $29 \mathrm{~s}$ ) correspond with spatial scales in the GSE $x$ direction on the order of an Earth radius (median: $\sim 4000 \mathrm{~km}$ ). These results are comparable to previous observations. Scale sizes and super-Alfvénic speeds of the HSJs suggest that reconnection cannot be a major generation mechanism of the observed jets.

8. Single spacecraft HSJ observation recurrence times are found to be on the order of a few minutes (median: $140 \mathrm{~s}$ ). However, even under favorable (low IMF cone angle) conditions, one spacecraft may not see any HSJs in prolonged magnetosheath intervals, although other spacecraft simultaneously present in the sheath may. Thus, we infer that HSJs are also localized in flow perpendicular dimensions.

9. The HSJ total pressure in the anti-sunward direction (toward the magnetopause) is enhanced by a factor of 2 , on average, due to large increases in dynamic pressure. Hence, the HSJs are expected to produce (large amplitude, but localized) magnetopause indentations, which are believed to result in magnetospheric compressional waves, magnetopause surface waves (Plaschke and Glassmeier, 2011), and/or ionospheric flow enhancements (Hietala et al., 2012).

Acknowledgements. We acknowledge NASA contract NAS502099 for use of data from the THEMIS Mission, specifically: C. W. Carlson and J. P. McFadden for the use of ESA data; K. H. Glassmeier, U. Auster and W. Baumjohann for the use of FGM data provided under the lead of the Technical University of Braunschweig and with financial support through the German Ministry for Economy and Technology and the German Center for Aviation and Space (DLR) under contract 50 OC 0302. The work of H. Hietala was funded by the UK Science and Technology Facilities Council (STFC)

Topical Editor C. Owen thanks two anonymous referees for their help in evaluating this paper. 


\section{References}

Amata, E., Savin, S. P., Ambrosino, D., Bogdanova, Y. V., Marcucci, M. F., Romanov, S., and Skalsky, A.: High kinetic energy density jets in the Earth's magnetosheath: A case study, Planet. Space Sci., 59, 482-494, doi:10.1016/j.pss.2010.07.021, 2011.

Angelopoulos, V.: The THEMIS Mission, Space Sci. Rev., 141, 534, doi:10.1007/s11214-008-9336-1, 2008.

Archer, M. O. and Horbury, T. S.: Magnetosheath dynamic pressure enhancements: occurrence and typical properties, Ann. Geophys., 31, 319-331, doi:10.5194/angeo-31-319-2013, 2013.

Archer, M. O., Horbury, T. S., and Eastwood, J. P.: Magnetosheath pressure pulses: Generation downstream of the bow shock from solar wind discontinuities, J. Geophys. Res., 117, A05228, doi:10.1029/2011JA017468, 2012.

Auster, H. U., Glassmeier, K. H., Magnes, W., Aydogar, O., Baumjohann, W., Constantinescu, D., Fischer, D., Fornacon, K. H., Georgescu, E., Harvey, P., Hillenmaier, O., Kroth, R., Ludlam, M., Narita, Y., Nakamura, R., Okrafka, K., Plaschke, F., Richter, I., Schwarzl, H., Stoll, B., Valavanoglou, A., and Wiedemann, M.: The THEMIS Fluxgate Magnetometer, Space Sci. Rev., 141, 235-264, doi:10.1007/s11214-008-9365-9, 2008.

Blanco-Cano, X., Omidi, N., and Russell, C. T.: Global hybrid simulations: Foreshock waves and cavitons under radial interplanetary magnetic field geometry, J. Geophys. Res., 114, A01216, doi:10.1029/2008JA013406, 2009.

Burgess, D., Lucek, E. A., Scholer, M., Bale, S. D., Balikhin, M. A., Balogh, A., Horbury, T. S., Krasnoselskikh, V. V., Kucharek, H., Lembège, B., Möbius, E., Schwartz, S. J., Thomsen, M. F., and Walker, S. N.: Quasi-parallel Shock Structure and Processes, Space Sci. Rev., 118, 205-222, doi:10.1007/s11214-005-3832-3, 2005.

Crooker, N. U., Siscoe, G. L., and Geller, R. B.: Persistent pressure anisotropy in the subsonic magnetosheath region, Geophys. Res. Lett., 3, 65-68, doi:10.1029/GL003i002p00065, 1976.

Czaykowska, A., Bauer, T. M., Treumann, R. A., and Baumjohann, W.: Magnetic field fluctuations across the Earth's bow shock, Ann. Geophys., 19, 275-287, doi:10.5194/angeo-19-275-2001, 2001.

Dmitriev, A. V. and Suvorova, A. V.: Traveling magnetopause distortion related to a large-scale magnetosheath plasma jet: THEMIS and ground-based observations, J. Geophys. Res., 117, A08217, doi:10.1029/2011JA016861, 2012.

Ellacott, S. W. and Wilkinson, W. P.: Heating of directly transmitted ions at low Mach number oblique collisionless shocks: A statistical physics formulation, Planet. Space Sci., 55, 2251-2256, doi:10.1016/j.pss.2007.05.018, 2007.

Glassmeier, K.-H. and Heppner, C.: Traveling magnetospheric convection twin vortices - Another case study, global characteristics, and a model, J. Geophys. Res., 97, 3977-3992, doi:10.1029/91JA02464, 1992.

Hietala, H. and Plaschke, F.: On the generation of magnetosheath high speed jets by bow shock ripples, J. Geophys. Res., submitted, 2013.

Hietala, H., Laitinen, T. V., Andréeová, K., Vainio, R., Vaivads, A., Palmroth, M., Pulkkinen, T. I., Koskinen, H. E. J., Lucek, E. A., and Rème, H.: Supermagnetosonic Jets behind a Collisionless Quasiparallel Shock, Phys. Rev. Lett., 103, 245001, doi:10.1103/PhysRevLett.103.245001, 2009.
Hietala, H., Partamies, N., Laitinen, T. V., Clausen, L. B. N., Facskó, G., Vaivads, A., Koskinen, H. E. J., Dandouras, I., Rème, H., and Lucek, E. A.: Supermagnetosonic subsolar magnetosheath jets and their effects: from the solar wind to the ionospheric convection, Ann. Geophys., 30, 33-48, doi:10.5194/angeo-30-33-2012, 2012.

Karlsson, T., Brenning, N., Nilsson, H., Trotignon, J.-G., Vallières, X., and Facsko, G.: Localized density enhancements in the magnetosheath: Three-dimensional morphology and possible importance for impulsive penetration, J. Geophys. Res., 117, A03227, doi:10.1029/2011JA017059, 2012.

Kataoka, R., Fukunishi, H., Lanzerotti, L. J., Maclennan, C. G., Frey, H. U., Mende, S. B., Doolittle, J. H., Rosenberg, T. J., and Weatherwax, A. T.: Magnetic impulse event: A detailed case study of extended ground and space observations, J. Geophys. Res., 106, 25873-25890, doi:10.1029/2000JA000314, 2001.

Kataoka, R., Fukunishi, H., and Lanzerotti, L. J.: Statistical identification of solar wind origins of magnetic impulse events, J. Geophys. Res., 108, 1436, doi:10.1029/2003JA010202, 2003.

King, J. H. and Papitashvili, N. E.: Solar wind spatial scales in and comparisons of hourly Wind and ACE plasma and magnetic field data, J. Geophys. Res., 110, A02104, doi:10.1029/2004JA010649, 2005.

Lin, Y., Lee, L. C., and Yan, M.: Generation of dynamic pressure pulses downstream of the bow shock by variations in the interplanetary magnetic field orientation, J. Geophys. Res., 101, 479494, doi:10.1029/95JA02985, 1996a.

Lin, Y., Swift, D. W., and Lee, L. C.: Simulation of pressure pulses in the bow shock and magnetosheath driven by variations in interplanetary magnetic field direction, J. Geophys. Res., 101, 27 251-27 270, doi:10.1029/96JA02733, 1996 b.

Lucek, E. A., Horbury, T. S., Dandouras, I., and RèMe, H.: Cluster observations of the Earth's quasi-parallel bow shock, J. Geophys. Res., 113, A07S02, doi:10.1029/2007JA012756, 2008.

McFadden, J. P., Carlson, C. W., Larson, D., Ludlam, M., Abiad, R., Elliott, B., Turin, P., Marckwordt, M., and Angelopoulos, V.: The THEMIS ESA Plasma Instrument and In-flight Calibration, Space Sci. Rev., 141, 277-302, doi:10.1007/s11214-008-9440-2, 2008.

Merka, J., Szabo, A., Slavin, J. A., and Peredo, M.: Threedimensional position and shape of the bow shock and their variation with upstream Mach numbers and interplanetary magnetic field orientation, J. Geophys. Res., 110, A04202, doi:10.1029/2004JA010944, 2005.

Němeček, Z., Šafránková, J., Přech, L., Sibeck, D. G., Kokubun, S., and Mukai, T.: Transient flux enhancements in the magnetosheath, Geophys. Res. Lett., 25, 1273-1276, doi:10.1029/98GL50873, 1998.

Omidi, N. and Sibeck, D. G.: Formation of hot flow anomalies and solitary shocks, J. Geophys. Res., 112, A01203, doi:10.1029/2006JA011663, 2007.

Omidi, N., Blanco-Cano, X., and Russell, C. T.: Macrostructure of collisionless bow shocks: 1. Scale lengths, J. Geophys. Res., 110, A12212, doi:10.1029/2005JA011169, 2005.

Plaschke, F. and Glassmeier, K.-H.: Properties of standing KruskalSchwarzschild-modes at the magnetopause, Ann. Geophys., 29, 1793-1807, doi:10.5194/angeo-29-1793-2011, 2011. 
Plaschke, F., Glassmeier, K.-H., Auster, H. U., Constantinescu, O. D., Magnes, W., Angelopoulos, V., Sibeck, D. G., and McFadden, J. P.: Standing Alfvén waves at the magnetopause, Geophys. Res. Lett., 36, L02104, doi:10.1029/2008GL036411, 2009.

Priest, E. and Forbes, T.: Magnetic Reconnection, Cambridge University Press, 2000.

Retinò, A., Sundkvist, D., Vaivads, A., Mozer, F., André, M., and Owen, C. J.: In situ evidence of magnetic reconnection in turbulent plasma, Nat. Phys., 3, 236-238, doi:10.1038/nphys574, 2007.

Savin, S. P., Zelenyi, L. M., Amata, E., Buechner, J., Blecki, J., Klimov, S. I., Nikutowski, B., Rauch, J. L., Romanov, S. A., Skalsky, A. A., Smirnov, V. N., Song, P., and Stasiewicz, K.: Dynamic interaction of plasma flow with the hot boundary layer of a geomagnetic trap, J. Exp. Theor. Phys. Lett., 79, 368-371, doi:10.1134/1.1772433, 2004.

Savin, S., Amata, E., Zelenyi, L., Budaev, V., Consolini, G., Treumann, R., Lucek, E., Safrankova, J., Nemecek, Z., Khotyaintsev, Y., Andre, M., Buechner, J., Alleyne, H., Song, P., Blecki, J., Rauch, J. L., Romanov, S., Klimov, S., and Skalsky, A.: High energy jets in the Earth's magnetosheath: Implications for plasma dynamics and anomalous transport, J. Exp. Theor. Phys. Lett., 87, 593-599, doi:10.1134/S0021364008110015, 2008.

Savin, S., Amata, E., Zelenyi, L., Lutsenko, V., Safrankova, J., Nemecek, Z., Borodkova, N., Buechner, J., Daly, P. W., Kronberg, E. A., Blecki, J., Budaev, V., Kozak, L., Skalsky, A., and Lezhen, L.: Super fast plasma streams as drivers of transient and anomalous magnetospheric dynamics, Ann. Geophys., 30, 1-7, doi:10.5194/angeo-30-1-2012, 2012.
Schwartz, S. J. and Burgess, D.: Quasi-parallel shocks - A patchwork of three-dimensional structures, Geophys. Res. Lett., 18, 373-376, doi:10.1029/91GL00138, 1991.

Schwartz, S. J., Paschmann, G., Sckopke, N., Bauer, T. M., Dunlop, M., Fazakerley, A. N., and Thomsen, M. F.: Conditions for the formation of hot flow anomalies at Earth's bow shock, J. Geophys. Res., 105, 12639-12650, doi:10.1029/1999JA000320, 2000.

Shue, J.-H., Song, P., Russell, C. T., Steinberg, J. T., Chao, J. K., Zastenker, G., Vaisberg, O. L., Kokubun, S., Singer, H. J., Detman, T. R., and Kawano, H.: Magnetopause location under extreme solar wind conditions, J. Geophys. Res., 103, 17691-17700, doi:10.1029/98JA01103, 1998.

Shue, J.-H., Chao, J.-K., Song, P., McFadden, J. P., Suvorova, A., Angelopoulos, V., Glassmeier, K. H., and Plaschke, F.: Anomalous magnetosheath flows and distorted subsolar magnetopause for radial interplanetary magnetic fields, Geophys. Res. Lett., 36, L18112, doi:10.1029/2009GL039842, 2009.

Turner, D. L., Omidi, N., Sibeck, D. G., and Angelopoulos, V.: First observations of foreshock bubbles upstream of Earth's bow shock: Characteristics and comparisons to HFAs, J. Geophys. Res., 118, 1552-1570, doi:10.1002/jgra.50198, 2013. 